Article

\title{
Revealing Motives for Car Use in Modern Cities-A Case Study from Berlin and San Francisco
}

\author{
Sascha von Behren ${ }^{1, * \mathbb{C}}$, Lisa Bönisch ${ }^{1}\left(\mathbb{D}\right.$, Ulrich Niklas $^{2}$ and Bastian Chlond ${ }^{1}$ \\ 1 Institute for Transport Studies, Karlsruhe Institute of Technology (KIT), 76131 Karlsruhe, Germany; \\ lisa.boenisch@kit.edu (L.B.); bastian.chlond@kit.edu (B.C.) \\ 2 BMW AG, 80788 Munich, Germany; ulrich.niklas@bmw.de \\ * Correspondence: sascha.vonbehren@kit.edu; Tel.: +49-0721-608-47736
}

Received: 9 April 2020; Accepted: 26 June 2020; Published: 29 June 2020

\begin{abstract}
Car use in modern cities with a well-developed public transit is more sophisticated to explain only through hard factors such as sociodemographic characteristics. In cities, it is especially important to consider motives for car use. Therefore, we examined two modern cities with a high modal share of non-motorized modes and public transit to answer the question: How do the affective and instrumental motives influence car use in such cities? The used data set was collected in Berlin and San Francisco. To investigate the role of motives, we applied an ordered hybrid choice model (OHCM) with a probit kernel. Based on the OHCM we explained more than $14 \%$ of the overall heterogeneity and gave further insights to the decision-making process. The affective motive had a strong influence on car use frequency, whereby the instrumental aspects did not matter. Furthermore, an effect resulting from age could not be determined for the affective motives in these cities. Results suggest people are more likely to use cars for affective motives despite the city's adversities. For these people it is difficult to achieve a shift to alternative means of transport. The only way to intervene here is through regulatory intervention.
\end{abstract}

Keywords: ordered hybrid choice model; car motives; travel skeleton; mobility culture

\section{Introduction}

The use of cars in cities is under discussion as it causes emissions and, moreover, disproportionately high consumption of urban space, both for driving and additionally for parking. Therefore, some cities have already introduced congestion charges to reduce traffic load (e.g., London or Stockholm). A discussed solution besides restrictions includes the substitution of private motorized transport by public transit ones. In particular in cities, an adequate offer of public transit is generally existing. However, detached from the availability of attractive mode alternatives and also from poor car use conditions due to congestion and parking problems, many people remain with the car use for their everyday travel. In this context, researchers and practitioners still question the reasons for the car being regarded as so attractive and the motives for its use, mainly in cities. A simple analysis of the figures confirms the current relevance of car trips. In Germany, for example, 36\% of all trips are done by cars in metropolitan areas [1]. Also, the annual mileage of private cars in urban areas is on average comparable with those of other spatial types [2]. In the field of transportation research, the influence of external factors like socio-demographic characteristic or travel time on car use have been investigated intensively. Besides external factors, existing literature refers to the important role of motives for car use. 


\subsection{Motives for Car Use}

The influence of soft factors, such as psychological factors, on travel behavior has been investigated and verified in a large number of studies. It has to be assumed that psychological factors also play a significant role in car use, which can be regarded as independent of the functional aspects and thus also of the existence and quality of other modes [3]. In general, people value car use positively, especially because of its flexibility, independence, availability, speed, reliability, safety, carrying capacity and comfort [4]. These subjective aspects address the apparent instrumental benefits of using cars for individual travel. Beyond this, for many people also in modern societies a car is positively associated by other psychological factors measured as a positive attitude towards using the car. A summary on various psychological correlates regarding car use and an extended overview of the literature has been given by Gardner and Abraham [5], who performed a meta-analysis with 23 unique datasets. In particular, motives for car use have become a widespread field of research. In this context, the designation of "car motives" is commonly used when psychological factors are considered together with car use.

Concerning car motives the multiple investigations of Steg $[4,6]$ are to be emphasized. She has found that the car is much more than just a means of transport to get from A to B. Car use is associated with strong affective and symbolic motives, even though daily experiences may not give reason [6]. As a result of her comprehensive research, she has finally provided an international accepted and well-tested set of psychological items that refers to three dimensions of motives for car use: instrumental, affective and symbolic. The affective value represents an experiential function of vehicles for individuals. Additionally, driving a car can be linked to positive emotions like driving pleasure. On the other hand, it can result in negative emotions because of stress while driving, e.g., due to traffic congestion [7]. The symbolic value of a car addresses its social impact in terms of a status symbol and the influence on social identity. These two additional psychological factors are likely to have a considerable impact on individual's emotions for car use [7]. It should be mentioned that the terminology related to car motives sometimes differs depending on the author, e.g., some use combinations such as "affective-symbolic" $[4,8,9]$ to describe the emotional value people attribute to their cars. Further investigations involving various psychological factors referring to peoples view on car travel also exist, e.g., Ellaway et al. [10], Shiftan et al. [11] and Anable [12], who have identified car-related behavior segments. The car-loving perspective has often been combined with environmental aspects, e.g., in the studies of Kamargianni et al. [13], Roberts et al. [14] or Hunecke et al. [15].

In particular, the item set of Steg [6] had been used as basis for related work of other authors who conducted more profound research on car use behavior [8,16-18]. Bergstadt et al. [8] have investigated the influence of socio-demographic variables on daily car use in Sweden, using OLS multiple linear regression analyses. They have found that affective-symbolic motives can partially describe the relationship between the number of weekly car trips and gender. In this context, they have been able to evidence that motives for car use partially explain differences in car use regarding different sociodemographic groups.

The reduction of private vehicle use, and the encouragement of public transit has always been the focus for practical implications of policy makers. When considering motives for car use against this background, we have to set our research into a wider context regarding availability of modes within cities and the reasons for people's use of theses modes. On one hand, people may use and value public transit for different motives than they do for car use. On the other hand, modal shift may be promoted by habitual behavior. In contrast to the low importance of the instrumental motive in terms of car use Nalmapantis et al. [19] found the opposite for public transit use. In their study feasibility and utility are the more important criteria regarding the use of public transit compared to the criterion of innovativeness. As a result, they suggest that people may increase their use of public transit if they notice an added value or direct impact for their travel. It seems that the practical benefit such as comfort or convenience is the more realistic motivation for people's behavior with regard to public 
transit use than aspects such as emotional excitement and the symbolic meaning. Indeed, this does not apply to car use, as affective motives play a strong role.

In general, most studies on car use consist of attitude-oriented models. For instance, Lois and López-Saez [16] used structural equation model (SEM) to examine the relation between affective, instrumental and symbolic motives. They have found that the affective motive has direct influence on car use, whereas symbolic and instrumental motives have only indirect influence through the affective motive. Their basic model was built up on three indirect variables measured as the three identified motives and the car use as latent variable. Twelve percent of the frequency of car use could be explained by people's affective link with their private car. Furthermore, applying SEM in their study, Sefara et al. [18] have shown that personal motives for using cars have a notable impact on the preferences for car types and brands. They have further concluded that for men the influence of affective-symbolic motives for car use have greater impact on car preference than for women.

\subsection{Motives for Car Use in Extended Discrete Choice Models}

Soft factors like motives are also increasingly used in discrete choice models. For this purpose, classical discrete choice models are extended with constructions of latent variables and have become popular under the name of hybrid choice model (HCM) [20-22]. These models are often also called integrated choice and latent variable (ICLV) models. However, in the following we use the term HCM. Examples for a provisional implementation in the case of mode choice are provided by Habib et al. [23], Yáñez et al. [24], Kamargianni et al. [13] and Johannsen et al. [25]. In view of the travel behavior investigated in recent research, it has to be summarized that the object of car use for commuting under consideration of psychological factors with this method has been studied extensively in the past $[14,17,25-27]$. Findings have confirmed the significance of psychological factors on the behavioral intention to commute by car. Some studies have focused on environmental aspects [14,27], but only few analyzed the impact of affective or symbolic motives on other trip purposes or car use frequency in general, e.g., Lois and López-Saez [16] or Bergstadt et al. [8]. However, they used other modeling techniques than HCM as mentioned above. Previous research have shown that the application of such hybrid choice models is clearly superior to classic choice models [13,24]. The advantage of the application of HCM lies in the model's ability to provide a more differentiated analysis regarding the influence of soft factors, such as motives, on people's behavior.

\subsection{Comparison of Car Use in Cities}

Besides the methodology of modeling technique also data base is a relevant aspect to consider when discussing the influence of affective motives on car use. Many of the stated literature have applied their research based on data from smaller cities or mixed residential locations $[8,14,28]$ or based on samples with special characteristics or small size $[13,16,17]$. However, especially the results of Bergstadt et al. [8] demonstrate higher ratings of affective-symbolic motives for car use for people living in metropolitan areas and with this evidenced the strong affective motive for car use from people in cities compared to other spatial types. For this reason, motives for car use in large cities, where many alternative mode options to the car exist, should be investigated more closely. In their conclusion some authors even directly have emphasized the necessity to conduct further research on the relevance of affective motives for people living in urban areas [8,28].

Considering international cultural differences Belgiawan et al. [9] have detected the symbolic-affective factor to be significant among attitudinal constructs in various countries when explaining the intention to purchase a car of undergraduate students. Further, Van and Fujii [29] have investigated and compared the contribution of psychological factors in explaining mode choice of students in six Asian countries. Their result indicates that attitude variables towards the car are significant determinants regardless of the country investigated. Apart from that, an international comparison of cities using an identical survey approach to collect adequate data regarding car use frequency in modern cities under consideration of instrumental and affective motives is still lacking. 


\subsection{Motivation and Scope of This Work}

In summary, an investigation of the soft factors influencing car use in modern cities with a high existing modal split share of public transit and non-motorized modes based on an identical survey approach and using an HCM is missing. The HCM is advantageous due to the fact that it takes utility maximization into account when considering the influence of soft factors, such as the psychological factors introduced. Moreover, in cities some people keep their cars only for occasional long-distance events and cars play a minor role in their everyday travel. The influences of long-distance travel activities of urbanites on the frequency of car use in everyday travel have been remained unconsidered by now. Concluding, the existing literature lacks in a holistic view of the influences of motives, long-distance transport activities of urbanites and spatial structure (e.g., availability of public transit) on car use. Research in this context plays a particularly relevant role in today's debate on car restrictions in cities due to affective motives of urban car users. Following this, our investigation has four main objectives:

- Discovering affective and instrumental motives for car use in modern cities;

- Applying a cost-efficient survey design to measure influences of long-distance travel behavior on everyday car use;

- Revealing the impact of population density and public transit supply on the frequency of car use;

- Giving insights in the decision-making process and explain more of the overall heterogeneity in pure regression model through the integration of motives in the HCM.

In order to consider these objectives, we required a comprehensive dataset that addresses all these issues. Therefore, we analyzed data from a survey conducted in the urban area of San Francisco (U.S.) and Berlin (Germany). The two selected cities are regarded as modern cities and have an identical mobility culture ("hybrid cities") [30]. Especially in cities the influence of soft factors on car use can be observed as many cities offer good access to public transit. Residents can choose between different modes of transport according to their individual preferences. Corresponding to this, the affective motive is gaining more weight when mode choice is considered there. To investigate the influence of motives for car use, we applied an HCM with an ordered probit kernel in the choice component. That means we used an ordered hybrid choice model (OHCM).

This study is structured as follows: first, we explain the concept of the travel skeleton and give information regarding the data collection; second, we describe the used data including the attitudinal constructs for the analysis; third, we explain the methodology of our analysis and the applied model specification; fourth, we present results of the $\mathrm{OHCM}$ and interpret resulting factors that influence car use in San Francisco and Berlin; finally, we draw a conclusion, discuss the limits of our approach and refer to further work.

\section{Concept of a Travel Skeleton and Data Collection}

The data used in this research is based on the concept of a travel skeleton. The idea of this concept derives from the longitudinal trip diary of the German Mobility Panel (MOP). Instead of asking the people about every single trip during a random week, we ask them about relevant activities and their mode choice in a "typical week" as a "pseudo-longitudinal" approach. Therefore, we capture the everyday travel through the important activities: work, leisure, chauffeuring, errands and shopping. Besides everyday travel this approach is also able to capture long-distance traveling, tech-savviness and psychological factors of respondents. The concept of a travel skeleton was originally designed and tested for a study in Hamburg and Berlin (Germany) [31]. For a more detailed description of the approach, we refer to von Behren et al. [32].

In the travel skeleton approach, we ask respondents, in detail about their typical car use. In addition to travel behavior, respondents are asked about the motives for car use. In our study, we focus on 13 psychological items (indicators), which are shown in Table 1. In addition, we also included items on public transit and cycling in the survey, which we had not used in this study. All items are taken from 
two standardized psychological item sets by Hunecke et al. [15] and Steg [6] and are rated on a Likert scale from 1-5 ("does not apply" to "apply").

Table 1. Psychological items (indicators) with their related questions.

\begin{tabular}{cc}
\hline Indicators & Psychological Questions \\
\hline$I_{1}^{A M}$ & I feel free and independent when I drive a car. \\
$I_{2}^{A M}$ & I like to drive a car. \\
$I_{3}^{A M}$ & Driving a car means fun and passion for me. \\
$I_{4}^{A M}$ & Driving a car means freedom to me. \\
$I_{5}^{A M}$ & Being able to use my driving skill when driving a car is fun for me. \\
$I_{6}^{A M}$ & When I sit in the car I feel safe and protected. \\
$I_{7}^{A M}$ & The make of a car is important to me. \\
$I_{1}^{I M}$ & The functioning of a car is more important to me than the make of a car. \\
$I_{2}^{I M}$ & A car is primarily a means to an end for me. \\
$I_{3}^{I M}$ & I only use a car to get from A to B. \\
$I_{4}^{I M}$ & It doesn't matter to me what vehicle type I drive. \\
$I_{5}^{I M}$ & I constantly have to be mobile in order to comply with my everyday obligations. \\
$I_{6}^{I M}$ & My everyday organization requires a high degree of mobility. \\
\hline
\end{tabular}

The presented research in this study was based on data collected through two similar surveys with face-to-face interviews, conducted in the urban area of Berlin (Germany) and San Francisco (U.S.) between October-2016 and January-2017. Both cities are comparable and regarded as "hybrid cities". The term "hybrid cities" describes a specific mobility culture. Kuhnimhof and Wulfhorst [33] explained mobility culture as a "set of values, conventions or social practices associated with the ability to travel from one point to another and with actual physical travel". As a result, city-wide attributes are addressed by the concept of urban mobility cultures, which include urban form and infrastructure supply as well as lifestyle and mobility patterns of a city's population [34]. In addition, hybrid cities exhibit dense public-transit-oriented urban cores with significant use of public transit and non-motorized modes, surrounded by low-density car-oriented suburban areas [30]. This is also reflected in the modal split of official statistics in the cities. In both cities, public transit has a share of more than $25 \%$ and walking more than $23 \%$ [35,36]. Concerning the cycling share San Francisco differs from Berlin, which can be explained by the topography. Both cities have comparable car use. San Francisco will change into an "e-hybrid city" over the medium-term (5-10 years) through the electrification of public and private transport [30].

The complete sample size was 1200 individuals with 600 respondents from each city. We conducted quota sampling regarding age, gender, household size and net income to develop a representative survey group for each captured city. For our analysis of the motives for car use, we used only the data of car users. As a result, we considered 836 people.

\section{Data Description and Statistical Analysis}

In our study, car use included not only the use of private cars. We also considered the use of car-sharing such as DriveNow, Car2go or Zipcar. The requirement, however, was that the person must drive the car himself. This also allowed us to consider the influence of car ownership on car use. For our model, respondent's choices were divided into three categories, according to the frequency of car use: daily use (day-to-day use of the car, 27\%, 225 respondents), weekly use (use of at least once up to a maximum of several times per week, 25\%, 205 respondents) and occasional use (use of less than once a week, $48 \%, 406$ respondents). Table 2 shows the characteristics of the persons surveyed and the variables subsequently used for the model. We included four dimensions in our analysis: sociodemographic characteristics, mobility and car use characteristics with regard to everyday travel and long-distance travel, and spatial form at the residential location. 
Table 2. Descriptive analysis of sociodemographic, mobility and spatial characteristics.

\begin{tabular}{|c|c|c|c|c|}
\hline Person Characteristics & & & & \\
\hline & Yes & No & & \\
\hline Age over 30 years & $78.23 \%$ & $21.77 \%$ & & \\
\hline Fulltime job & $57.30 \%$ & $42.70 \%$ & & \\
\hline Male & $52.27 \%$ & $47.73 \%$ & & \\
\hline \multirow[t]{2}{*}{ Own bicycle } & $48.92 \%$ & $51.08 \%$ & & \\
\hline & Always & Sometimes & Never & \\
\hline Share of car disposal & $46.66 \%$ & $23.20 \%$ & $30.14 \%$ & \\
\hline \multicolumn{5}{|l|}{ Household Characteristics } \\
\hline & Yes & No & & \\
\hline Premium car in household & $20.45 \%$ & $79.55 \%$ & & \\
\hline Household from Berlin & $47.73 \%$ & $52.27 \%$ & & \\
\hline \multirow[b]{2}{*}{ Share of income classes } & $<\$ 2500$ & $\$ 2500-\$ 5000$ & $\$ 5001-\$ 8000$ & $>\$ 8000$ \\
\hline & $16.75 \%$ & $32.54 \%$ & $31.34 \%$ & $24.76 \%$ \\
\hline \multirow[b]{2}{*}{ Share of household type } & Type 1 & Type 2 & Type 3 & Type 4 \\
\hline & $31.22 \%$ & $18.30 \%$ & $25.72 \%$ & $24.76 \%$ \\
\hline \multicolumn{5}{|c|}{ Mobility and Car Use Characteristics } \\
\hline & Yes & No & & \\
\hline Commuting by car & $73.09 \%$ & $26.91 \%$ & & \\
\hline Monomodal behavior & $29.19 \%$ & $70.81 \%$ & & \\
\hline Large number of daytrips & $4.07 \%$ & $95.93 \%$ & & \\
\hline Large number of vacation trips & $8.73 \%$ & $91.27 \%$ & & \\
\hline Long-distance trips by car & $23.56 \%$ & $76.44 \%$ & & \\
\hline \multicolumn{5}{|l|}{ Spatial Characteristics } \\
\hline \multirow{4}{*}{$\begin{array}{l}\text { High population density } \\
\text { High public transit accessibility }\end{array}$} & Yes & No & & \\
\hline & $39.71 \%$ & $60.29 \%$ & & \\
\hline & $25.84 \%$ & $74.16 \%$ & & \\
\hline & & & & $\mathrm{N}=83$ \\
\hline
\end{tabular}

Three classes were created to describe access to the car in the household. The variable "car disposal-sometimes" describes whether the person can only access the car by agreement in the household. "Car disposal-always" describes a permanent access to a car in the household. For 47\% of the respondents, no agreement within the household was necessary to use the private car (see Table 2). Twenty percent of the households in our sample disposed on at least one premium car from brands such as BMW, Mercedes, Audi or Tesla. We further analyzed if persons in general used a car for commuting (73\%). Twenty-nine percent of our respondents had a monomodal behavior, i.e., they used the same mode of transport in everyday travel. Table 2 shows the share of people with a large number of daytrips or vacation trips with overnight stays. A high number of daytrips means more than 12 trips per year and a high number of vacation trips describe more than eight trips per year. In our sample, $24 \%$ of the respondents used a car for long-distance leisure trips over $100 \mathrm{~km}$. For the evaluation of car use it is important to also consider the spatial structure. Therefore, we included two spatial information on zip code level regarding the residential location of the respondents. On one hand, we used open street map (OSM) data to calculate the built-up area and data about the population from the data provider Nexiga to calculate the population density per built-up area. High population density includes all zip codes with more than 10,000 inhabitants per built-up area per square meter. On the other hand, we calculated the public transit options and quality in the zip codes under the use of OSM data. When calculating the accessibility, we considered that rail-bound public transit is more valuable than buses. A high calculated value indicates people have plenty of public transit options besides the car in this zip code. This applied to $26 \%$ of the respondents.

\section{Attitudinal Constructs}

In addition to the variables mentioned above, we also considered psychological factors for our analysis. The answers to the attitudinal questions are summarized in Figure 1. In the case of the 
indicators describing affective motives of driving $\left(I_{1-6}^{A M}\right)$, almost $50 \%$ of the participants agreed on all the questions. For the item $I_{7}^{A M}$ only $37 \%$ of the participants agreed. Over $60 \%$ agreed with the statement: "I like to drive a car" $\left(I_{2}^{A M}\right)$, where the question is general. However, for more than $30 \%$ driving a car does not mean fun or passion $\left(I_{3}^{A M}\right)$. As can be seen, roughly more than $50 \%$ of the respondents rated the instrumental motives $\left(I_{1-3}^{I M}\right)$ positively. Especially for nearly $70 \%$ the function of a car was more important than the make $\left(I_{1}^{I M}\right)$.

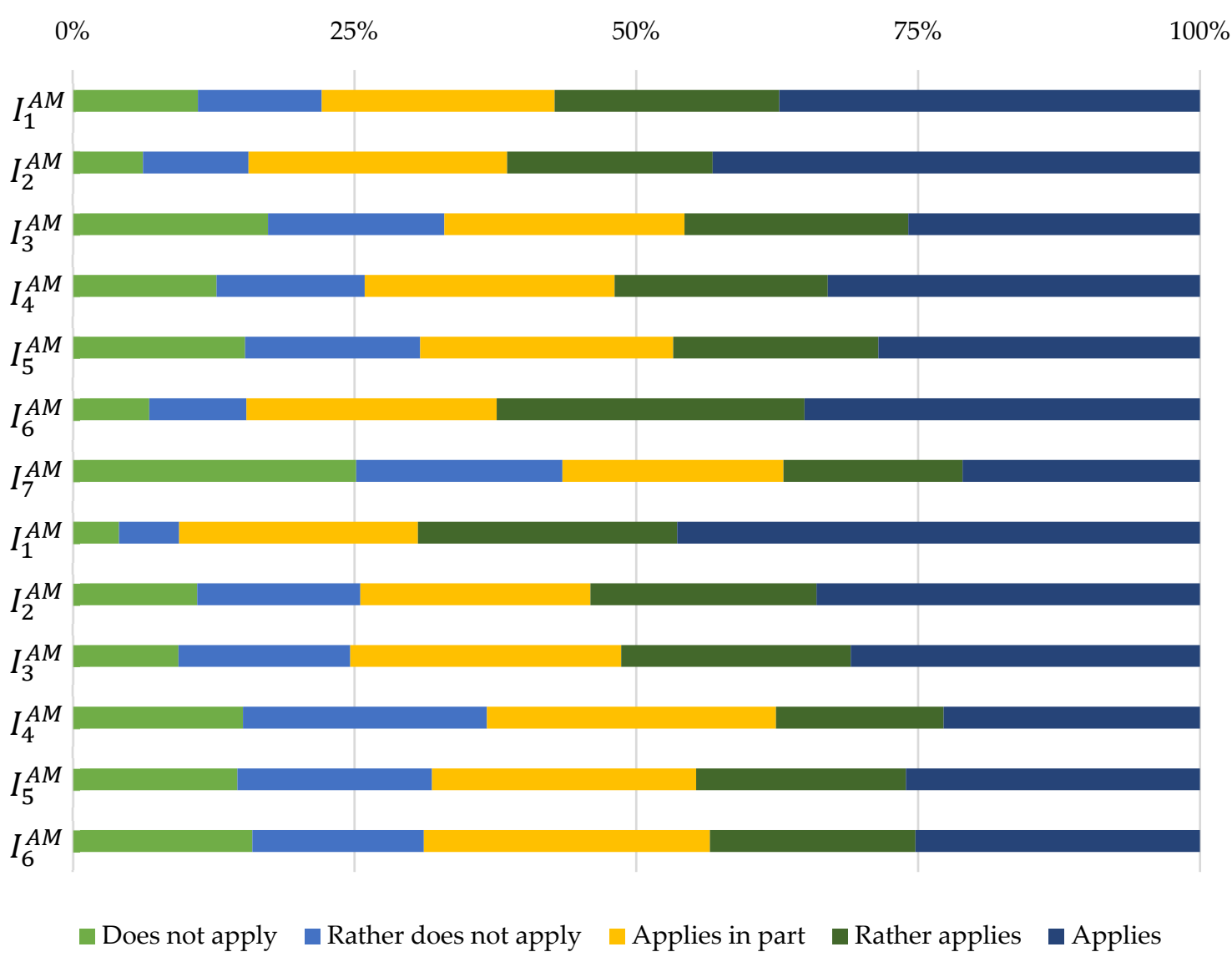

Figure 1. Motives for car use. The 1-5 Likert-scaled questions as described in Table 1.

As a preliminary analysis to our ordered hybrid choice model (OHCM) and to identify latent variables, we conducted an explorative factor analysis based on the presented attitudinal indicators. Table 3 shows the result of the principal factor analysis (PFA) with varimax rotated factor pattern. Based on the scree plot (elbow criterion) and the Kaiser's criterion, two factors could be extracted: one describing the affective motive $\left(I_{1-7}^{A M}\right)$ and one describing the instrumental motive $\left(I_{1-4}^{I M}\right)$ of car use. The two items $I_{5}^{I M}$ and $I_{6}^{I M}$ are not considered further due to their low factor loadings on the two factors. The factor "affective motive" (AM) describes whether people like to drive a car and whether they feel free through the use. The factor "instrumental motive" (IM) describes whether people only use the car as a tool to satisfy their mobility needs. Both factors were found to have sufficient internal consistency (reliability) with a Cronbach's alpha of 0.90 (excellent) for AM and 0.72 (acceptable) for IM, respectively. The low value for alpha of IM (0.72) showed only an acceptable reliability due to the number of items for the measure. This is sufficient but must be considered for the further analysis. 
Table 3. Principal factor analysis (PFA).

\begin{tabular}{crr}
\hline \multicolumn{3}{c}{ Factors } \\
\hline \multicolumn{4}{c}{ Affective Motive (AM) } & Instrumental Motive (IM) \\
\hline Indicators in PFA & $\mathbf{0 . 8 4 4 9 6}$ & \\
$I_{1}^{A M}$ & $\mathbf{0 . 8 2 7 9 4}$ & 0.04663 \\
$I_{2}^{A M}$ & $\mathbf{0 . 8 0 9 5 2}$ & 0.08091 \\
$I_{4}^{A M}$ & $\mathbf{0 . 7 6 7 3 8}$ & 0.01878 \\
$I_{5}^{A M}$ & $\mathbf{0 . 7 6 4 6 3}$ & 0.07639 \\
$I_{3}^{A M}$ & $\mathbf{0 . 6 2 8 1 5}$ & -0.04576 \\
$I_{6}^{A M}$ & $\mathbf{0 . 5 4 7 5 5}$ & -0.01329 \\
$I_{7}^{A M}$ & -0.03506 & -0.23227 \\
$I_{3}^{I M}$ & -0.13355 & $\mathbf{0 . 6 9 4 4 9}$ \\
$I_{2}^{I M}$ & 0.09295 & $\mathbf{0 . 6 4 4 2 8}$ \\
$I_{1}^{I M}$ & -0.15331 & $\mathbf{0 . 5 8 6 1 2}$ \\
$I_{4}^{I M}$ & 0.21989 & $\mathbf{0 . 4 9 2 2 2}$ \\
$I_{6}^{I M}$ & 0.23032 & 0.27218 \\
$I_{5}^{I M}$ & High factor loadings & 0.26416 \\
\hline
\end{tabular}

\section{Methodology Approach}

In our applied models, individual's main choice was the regularity of car use on an ordered scale (daily/weekly/occasional use). This choice was modeled through an ordered probit model (ORP). We calculated this model as a "base" model prior to the ordered hybrid choice model (OHCM). Therefore, we selected a probit model over a logit model to construct an identifiable reduced form model. In this section, we focus on the OHCM. For more information about ORP, we refer to further literature.

\subsection{Ordered Hybrid Choice Model}

Thus, far, in the "base" model we only considered observable variables to describe the choices. The OHCM provides a framework to incorporate unobservable, latent variables. In the following, we describe the setting of the OHCM that is later being used. The indicators Table 1 were used to gain information on the latent variables describing people's motive for car use. By adding a latent variable model to the choice component, we were able to include these indicators. As a result, the model contained a group of structural equations and measurement equations. In the following, these equations are described in more detail.

In the OHCM, the vector of the $L$ different latent variables of individual $n$ is described by $Z_{n l}$. Each latent variable $Z_{n l}$ is a linear combination of the individual's observable variables $X_{n}$ and a random component $\xi_{n l}$, which is assumed to be normally distributed with mean zero and variance $\sigma_{l}^{2}$. The vectors $\alpha_{l}$ are the weights in the structural equation. Thus, the following equation for the $l$-th latent variable is obtained:

$$
Z_{n l}=\alpha_{l} X_{n}+\xi_{n l}, \xi_{n l} \sim N\left(0, \sigma_{l}^{2}\right) .
$$

In our model, we assumed independency of the latent variables given the observable variables and the weights $\alpha_{l}$. The random component $\xi_{n l}$ is assumed to be independent for all $l \in\{1, \ldots, L\}$ and $n \in\{1, \ldots, N\}$. As scale and spread of the latent variables are arbitrary, no constant is needed in the specification of the latent variables and the variance can be set to one.

The indicators are manifestations of the latent variables. The responses of these indicators are given on an ordered scale and represented by the values 1 to $S$. We indicated the set of indicators of individual $n$ by the vector $I_{n}$, consisting of the $K$ single indicators $I_{n k}, k \in\{1, \ldots, K\}$. Each indicator is 
modeled by a continuous representation $\tilde{I}_{n k}$. The indicators $I_{n k}$ are composed of a linear combination of the latent variables with weights $\zeta_{k}$ and an error component $\psi_{n k}$ with mean zero:

$$
\tilde{I}_{n k}=\zeta_{k} Z_{n}+\psi_{n k}
$$

The distribution of the Indicators $I_{n k}$ is then defined by allocating the variables $\tilde{I}_{n k}$ to intervals given by the thresholds $\rho_{k}^{(0)}, \ldots, \rho_{k}^{(S)}$ :

$$
\begin{array}{ccc}
I_{n k}=1 & \Leftrightarrow \quad \rho_{k}^{(0)}<\tilde{I}_{n k} \leq \rho_{k}^{(1)}, \\
I_{n k}=2 & \Leftrightarrow \quad \rho_{k}^{(1)}<\tilde{I}_{n k} \leq \rho_{k}^{(2)}, \\
& \vdots & \\
I_{n k}=S & \Leftrightarrow & \rho_{k}^{(S-1)}<\tilde{I}_{n k} \leq \rho_{k}^{(S)},
\end{array}
$$

where $\rho_{k}^{(0)}=-\infty$ and $\rho_{k}^{(S)}=\infty$ for all $k \in\{1, \ldots, K\}$. The error components $\psi_{n k}$ are i.i.d. and follow a Gumbel distribution for all $n$ and $k$. We obtain an ordered logit model to explain the likelihood of the observed values of $I_{n k}$ of individual $n$ as:

$$
\begin{aligned}
P\left(I_{n k}=S \mid Z_{n}, \zeta_{k}\right) & =P\left(\rho_{k}^{(s-1)}<\tilde{I}_{n k} \leq \rho_{k}^{(s)} \mid Z_{n}, \zeta_{k}\right) \\
& =\frac{\exp \left(\rho_{k}^{(s)}-\zeta_{k} Z_{n}\right)}{1+\exp \left(\rho_{k}^{(s)}-\zeta_{k} Z_{n}\right)}-\frac{\exp \left(\rho_{k}^{(s-1)}-\zeta_{k} Z_{n}\right)}{1+\exp \left(\rho_{k}^{(s-1)}-\zeta_{k} Z_{n}\right)^{\prime}}
\end{aligned}
$$

In the utility function of the choice component, we incorporated the latent variables $Z_{n}$ analogously to the observable variables $X_{n}$ :

$$
U_{n}=\beta X_{n}+\gamma Z_{n}+\epsilon_{n}
$$

where $\gamma$ are the weights of the latent variables $Z_{n}$. The choice probabilities in the OHCM, given all variables $X_{n}$ and $Z_{n}$, is then calculated as in the ORP:

$$
P\left(Y_{n}=j \mid X_{n}, Z_{n}, \beta, \gamma, \tau\right)=\Phi\left(\frac{\tau^{(j)}-\left(\beta X_{n}+\gamma Z_{n}\right)}{\sigma_{\text {Choice }}}\right)-\Phi\left(\frac{\tau^{(j-1)}-\left(\beta X_{n}+\gamma Z_{n}\right)}{\sigma_{\text {Choice }}}\right) .
$$

The error component $\epsilon_{n}$ contains all unobserved heterogeneity. By integrating the latent variables, which are random variables themselves, as in Equation (5) we attempted to reduce the role of the purely random terms $\epsilon_{n}$. We were able to shift unobserved heterogeneity of the error component to observed heterogeneity through the latent variables. Kamargianni et al. [13] used also HCMs with a probit kernel, but they applied a multinomial probit model. They were inspired by Bhat and Dubey [37], who have proposed the probit kernel over the logit kernel in their work.

Based on the described OHCM specification, the likelihood can now be calculated for given data and parameters (consisting of $\beta, \gamma, \tau, \sigma, \zeta, \rho, \alpha$ ). Thus, values for the parameters can be estimated using the maximum likelihood method. However, the OHCM requires the evaluation of $L$ dimensional integrals due to the $L$ different latent variables, which is intractable. We approximated these integrals using draws (e.g., Halton draws) according to the distribution of the latent variables, resulting in the maximum simulated likelihood estimation method.

\subsection{Construct of Reduced Form Model}

Based on the probit model, we constructed a reduced form model of the OHCM to compare the results with the "base" model. Therefore, we applied the approach of Vij and Walker [38]. Inserting the Equation (1) of the latent variables $Z_{n}$ in the utility function (Equation (5)), we received 


$$
\begin{aligned}
U_{n} & =\beta X_{n}+\gamma Z_{n}+\epsilon_{n} \\
& =\beta X_{n}+\gamma_{1}\left(\alpha_{1} X_{n}+\xi_{n 1}\right)+\cdots+\gamma_{L}\left(\alpha_{L} X_{n}+\xi_{n L}\right)+\epsilon_{n} \\
& =\left(\beta+\gamma_{1} \alpha_{1}+\cdots+\gamma_{L} \alpha_{L}\right) X_{n}+\gamma_{1} \xi_{n 1}+\cdots+\gamma_{L} \xi_{n L}+\epsilon_{n} \\
& =\beta_{R F M} X_{n}+\epsilon_{R F M}
\end{aligned}
$$

with $\beta_{R F M}=\beta+\gamma_{1} \alpha_{1}+\cdots+\gamma_{L} \alpha_{L}$ and $\epsilon_{R F M}=\gamma_{1} \xi_{n 1}+\cdots+\gamma_{L} \xi_{n L}+\epsilon_{n}$. The error components $\xi_{n l}, l \in\{1, \cdots, L\}$ of the latent variables are all independently standard normally distributed. Therefore, the products of $\gamma_{l} \xi_{n l}$ are independently normally distributed with variance $\gamma_{l}^{2}$. Given that, we used an ordered probit (ORP) model in the choice component, i.e., the error component $\epsilon_{n}$ of utility is also independently normally distributed with variance $\sigma^{2}, \epsilon_{R F M}$ is a sum of $L+1$ independent normally distributed random variables, and thus again a normally distributed error component with variance $\sigma_{R F M}^{2} \gamma_{1}^{2}+\cdots+\gamma_{L}^{2}+\sigma^{2}$. Overall, the OHCM is again an ORP model with the aggregated weights $\beta_{R F M}$ for the observable variables and with variance $\sigma_{R F M}^{2}$. As a consequence, we can expect only that the choice component of the OHCM is as good as an ORP model in terms of the overall likelihood value, when using identical variables. The reason is that in the "base" ORP model the parameters are only estimated to describe the choices for the alternatives as good as possible, whereas in the OHCM the parameters are estimated to describe the choices for the alternatives simultaneously with the responses to the indicators. Ideally, the choices in the OHCM are described in the same manner and further insights to the decision-making process are provided.

\section{Applied Model Specification of Motives for Car Use}

The ordered hybrid choice model (OHCM) was defined according to the equations in the methodology section. Figure 2 shows the final model specification. After extensive specification testing in the "base" ordered probit (ORP) model, we inserted the explanatory variables in our OHCM as shown in Figure 2. This OHCM was based on a pooled dataset with respondents from San Francisco and Berlin to identify influences on car use in modern cities. For our model, we created a holistic view on influences considering the following aspects:

- sociodemographic characteristics (person and household);

- long-distance travel activities;

- everyday travel;

- $\quad$ spatial structure at residential location;

- motives for car use.

The following variables were used as dummy variables in the model specification (as described in the section descriptive results): age, fulltime job, male, premium car in household, from Berlin, daytrips, vacation trips, commuting by car, long-distance trips by car, monomodal, population density and public transit accessibility. The city dummy variable "from Berlin" captures all factors excluded from the analysis-such as culture and policy (besides the identical mobility culture) as well as spatial variables (others than density and public transit quality). The dummy variable low income describes people with a monthly household net income under $\$ 2500$. We also included the car disposal (split into three categories, taking never as the base) and the household type (split into three categories, taking household type 1 and 2 as one- or two-person households as the base). Consequently, $18 \beta$-parameters and two $\gamma$-parameters for the influence of the variables on utility, the parameter $\sigma_{\text {choice }}^{2}$ describing the variance of the error component in the utility, $14 \alpha$-parameters for the structural equations of the latent variables affective and instrumental motive, respectively, as well as the parameters $\zeta$ and $\rho$ for the indicators had to be estimated. In total we had to estimate 93 parameters. To set the scale of utility, one of the parameters included in the specification of utility had to be fixed. Usually, this is done by setting the variance $\sigma_{\text {choice }}^{2}$ to one, but we decided to fix one of the $\beta$ parameters instead, such that we could compare the variance of the purely random component in the OHCM and in the reduced form model in the end. Ultimately, the parameter for the variable describing if people use a 
car for commuting was set to one. For estimation, we adapted the code of the Choice Modelling Center (CMC) for R [39] with the application of an ordered probit kernel and used 5000 Halton draws for each latent variable.

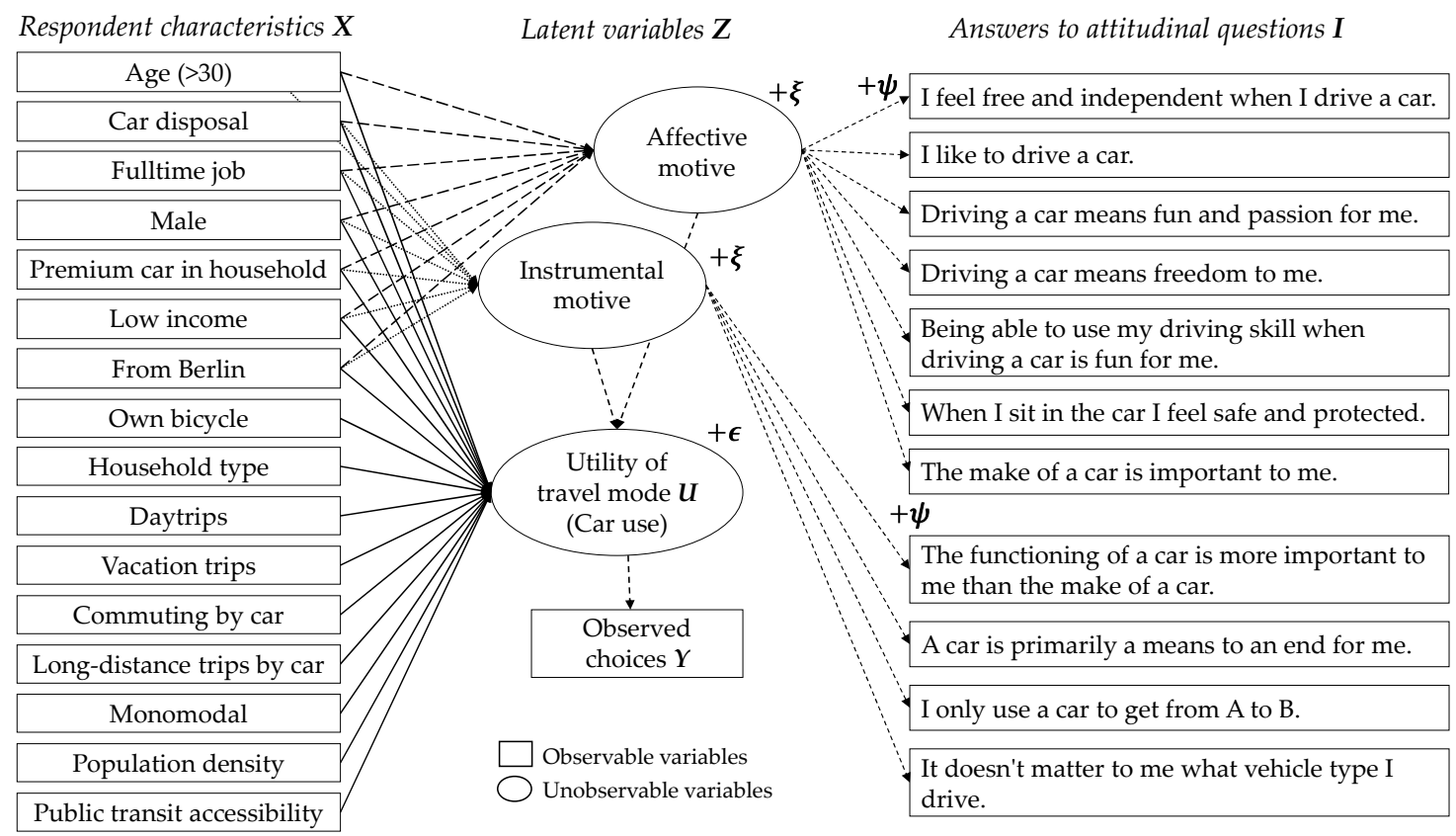

Figure 2. Specification of the ordered hybrid choice model (OHCM).

\section{Results of the Ordered Hybrid Choice Model}

In this section, we first discuss the influence of the respondents' characteristics on the affective and instrumental motive (structural model). Second, we consider and interpret the direct influences of the respondents' characteristics and the motives on car use (see Figure 2). Last, we assess the overall effects of the ordered hybrid choice model (OHCM) and compare them with the "base" ordered probit (ORP) model.

\subsection{Effects on Instrumental and Affective Motives}

Table 4B presents the findings regarding the effects of observable variables on instrumental and affective motives. For young car users a higher value for affective motives and a lower value for instrumental motives was observed. Among older people a higher influence of the instrumental motive was found. This is in contrast to findings of Van and Fujii [29] and Vos and Alemi [28] and implies that if young adults use a car, they will have stronger affective motives than older ones. We saw that the younger generation is not independent of cars, even in modern cities. Further, persons from households with premium cars had higher affective motives and lower instrumental motives than persons without a premium car in their household. This finding could be confirmed indirectly by the work of Sefara et al. [18] who have determined the impact of personal motives on preferences regarding car type. In addition, a higher instrumental motive was observed among people from Berlin. But we could not see any influence regarding the origin on the affective motive. 
Table 4. Main parameter estimates. (A) Parameters of the structural equation of the choice model; (B) Parameters of the structural equations of the latent variables.

\begin{tabular}{|c|c|c|c|c|c|c|c|}
\hline \multicolumn{4}{|c|}{$\begin{array}{c}\text { Log-likelihood } \\
\text { Log-likelihood of choice component } \\
\text { Log-likelihood of null model (choice component) } \\
\text { McFadden pseudo- } R^{2}\end{array}$} & & & $\begin{array}{l}116.04 \\
57.26 \\
18.44 \\
.39\end{array}$ & \\
\hline \multicolumn{4}{|c|}{$\mathrm{N}$} & \multicolumn{4}{|c|}{836} \\
\hline \multicolumn{8}{|c|}{ Thresholds of the choice component } \\
\hline$\tau^{(1)}$ & \multicolumn{7}{|c|}{1.814} \\
\hline$\tau^{(2)}$ & \multicolumn{7}{|c|}{3.075} \\
\hline \multicolumn{3}{|c|}{ (A) } & \multicolumn{5}{|c|}{ (B) } \\
\hline \multirow{2}{*}{ Parameter } & \multirow{2}{*}{\multicolumn{2}{|c|}{ Value }} & \multirow{2}{*}{ Parameter $\alpha$ from variable } & \multicolumn{4}{|c|}{ on latent variable } \\
\hline & & & & \multicolumn{2}{|c|}{ affective motive } & \multicolumn{2}{|c|}{ instrumental motive } \\
\hline$\beta_{\text {age }>30}$ & 0.472 & $* * *$ & Age $>30$ years & -0.194 & $* *$ & 0.172 & * \\
\hline$\beta_{\text {fulltime }}$ & 0.148 & & Fulltime job & 0.210 & $* * *$ & 0.079 & \\
\hline$\beta_{\text {male }}$ & 0.003 & & Male & 0.398 & $* * *$ & -0.375 & $* * *$ \\
\hline$\beta_{\text {bicycle }}$ & -0.078 & & & & & & \\
\hline$\beta_{\text {car-sometimes }}$ & 0.183 & & Car disposal - sometimes & 0.660 & $* * *$ & 0.286 & ** \\
\hline$\beta_{\text {car-always }}$ & 1.214 & $* * *$ & Car disposal - always & 1.126 & $* * *$ & 0.294 & $* * *$ \\
\hline$\beta_{\text {premiumcar }}$ & 0.235 & $* *$ & Premium car in householc & 0.664 & $* * *$ & -0.035 & \\
\hline$\beta_{\text {berlin }}$ & 0.097 & & From Berlin & 0.014 & & 0.980 & $* * *$ \\
\hline$\beta_{\text {lowincome }}$ & -0.322 & $* *$ & Low income & 0.308 & $* * *$ & 0.290 & $* *$ \\
\hline$\beta_{\text {hhtype3 }}$ & 0.320 & $* * *$ & & & & & \\
\hline$\beta_{\text {hhtype4 }}$ & 0.200 & * & & & & & \\
\hline$\beta_{\text {highdaytrips }}$ & 0.533 & $*$ & & & & & \\
\hline$\beta_{\text {highvacation }}$ & -0.085 & & & & & & \\
\hline$\beta_{\text {commuting_car }}$ & 1.000 & & & & & & \\
\hline$\beta_{\text {long-distance_car }}$ & 0.304 & $* * *$ & & & & & \\
\hline$\beta_{\text {monomodal }}$ & -0.194 & * & & & & & \\
\hline$\beta_{\text {density }}$ & -0.047 & & & & & & \\
\hline$\beta_{p t \_a c c e s s i b i l i t y}$ & -0.112 & & & & & & \\
\hline$\gamma_{\text {affective }}$ & 0.398 & $* * *$ & & & & & \\
\hline$\gamma_{\text {instrumental }}$ & 0.020 & & & & & & \\
\hline$\sigma$ & 0.970 & $* * *$ & & & & & \\
\hline
\end{tabular}

Parameters marked with ${ }^{* * *},{ }^{* *}$ and * are significant at the $1 \%, 5 \%$ and $10 \%$ level, respectively.

\subsection{Direct Effects on Car Use}

The OHCM helps to illustrate the influence of various socio-demographic characteristics, long-distance travel behavior, spatial forms as well as motives on car use frequency. For the analysis, we first look at the direct impact of the respondents' characteristics on car use frequency (see Table 4A). The high influence of age was not surprising, showing people over the age of 30 were more likely to use a car more often. The highest influence on car use could be traced back to car availability. People who had permanent access to a car in their household were more likely to use it. We cannot see this high influence if the person could only use a car in agreement with the household. A premium car in the household also increased the frequency of car use. This is in line with expectations and is also confirmed in the study on the use of premium vehicles compared to conventional ones done by Niklas et al. [40]. The results in the OHCM show that car use is not influenced directly by the place of residence: car users from Berlin did not use a car more often than users from San Francisco. If there was a significant difference in car use, an impact would have been visible here. Furthermore, our results show a higher probability for car use in everyday travel when people reported many daytrips at the weekend. The reverse effect was observable for people conducting a considerable number of overnight stays. In San Francisco and Berlin, people with lower incomes used a car less frequently than people from higher income groups. With reference to monomodal behavior, people who used rather few different means of transport were also less likely to use a car more often. This is surprising, as frequent car users in particular are monomodal persons. In the case of spatial structures, an influence from the 
offer of public transit services could be recognized. People used cars less frequently if alternatives such as public transit exist. Consequently, these people are less dependent on cars.

In a further step, we look at the impact of the affective and the instrumental motive on car use frequency (see Table 4A). As a result, we see a significant positive influence of the affective motive. Respondents with a high value regarding the affective motive showed a greater utility for car use. In contrast, we detected no influence of the instrumental motive. This result is in line with existing research about motives [16]. It is interesting that this result also applies to modern cities like Berlin and San Francisco. On this basis, we assume that the instrumental motive is diminished by the good public transit services or the availability of on-demand mobility services like ride hailing. The influence of the affective motive complicates the substitution of the car through other means of transport, as the reduction in car use cannot be solved on the supply side.

\subsection{Overall Effects on Car Use}

The OHCM provides the advantage of allowing us to examine the split into direct effects of the observable variables and effects through the LVs. Therefore, we constructed a reduced form model of the OHCM. The direct influences and the influences through the LVs on the choice to use a car are summarized in Table 5. By looking at the affective motive, it becomes clear that the influence of gender exclusively arose through this motive. Consequently, men with a higher affective motive were more likely to use cars. Further, results show an unmediated influence of age on car use, but we could not observe an age effect through the affective motive. People over the age of 30 years did not use a car more often due to higher affective motives. We suggest that they may have other mobility requirements compared to younger people. In addition, the influences of car availability and the presence of a premium vehicle in the household were reinforced by the affective motive. The effect of the premium vehicle and the availability through agreements in the household was even doubled through the affective motive. As we have already seen in Table 4A, a low income had a negative effect on car use. However, this effect is reduced if the person had an affective motive for car use. This suggests that people dispense with other things to be able to use a car, even if it is more expensive than public transit or cycling. In the overall effect of the OHCM, we can see a low not significant influence of Berlin on the car use frequency.

Table 5. Direct influences vs. influences through the latent variables (LVs) on the choice.

\begin{tabular}{|c|c|c|c|c|c|c|}
\hline Variable & Direct Effect & $\begin{array}{r}\text { Effect } \\
\text { via LV } \\
\text { Affective Motive }\end{array}$ & $\begin{array}{r}\text { Effect } \\
\text { via LV Instrumental } \\
\text { Motive }\end{array}$ & $\begin{array}{r}\text { Effect } \\
\text { via LVs } \\
\text { Combined }\end{array}$ & Overall, Effect & $\begin{array}{l}\text { Overall, Effect in } \\
\text { Ordered Probit }\end{array}$ \\
\hline Age $>30$ years & 0.472 & -0.077 & 0.003 & -0.074 & 0.398 & 0.347 \\
\hline Male & 0.003 & 0.158 & -0.007 & 0.151 & 0.154 & 0.139 \\
\hline Own bicycle & -0.078 & & & & -0.078 & -0.146 \\
\hline Car disposal - sometimes & 0.183 & 0.262 & 0.006 & 0.268 & 0.451 & 0.355 \\
\hline Car disposal - always & 1.214 & 0.448 & 0.006 & 0.454 & 1.668 & 1.414 \\
\hline Low income & -0.322 & 0.122 & 0.006 & 0.128 & -0.194 & -0.157 \\
\hline Household type 3 & 0.320 & & & & 0.320 & 0.233 \\
\hline Household type 4 & 0.200 & & & & 0.200 & 0.228 \\
\hline High daytrips & 0.533 & & & & 0.533 & 0.559 \\
\hline High vacation trips & -0.085 & & & & -0.085 & -0.135 \\
\hline Commuting by car & 1.000 & & & & 1.000 & 1.000 \\
\hline Long-distance trips by car & 0.304 & & & & 0.304 & 0.305 \\
\hline
\end{tabular}

By reviewing the two last columns in Table 5, we see that the overall effects of the observable variables on car use frequency in the OHCM are, as expected, almost identical to the effects in a separately estimated "base" ORP model. This also applies to the threshold values. The overall variance in the OHCM is almost identical to the variance in the pure ORP model. This result provides a strong 
confidence in the parameter estimates in our OHCM. Small deviations are to be expected due to simulation noise when using the maximum simulated likelihood method with Halton draws.

A relevant aspect of integrating latent variables in the OHCM is the aim to explain some of the unobserved heterogeneity. Therefore, we analyzed the share of heterogeneity in the model that can actually be linked to the LVs. The share was thus

$$
\frac{\gamma_{\text {affective }}^{2}+\gamma_{\text {instrumental }}^{2}}{\sigma^{2}+\gamma_{\text {affective }}^{2}+\gamma_{\text {instrumental }}^{2}}=14.43 \% .
$$

By adding the motives to the model, we were able to explain a large part of the unexplained heterogeneity in the "base" ORP model. In addition to the insights gained, this argues for the application of this model approach to clarify questions of this type. The limitation is the significantly higher calculation effort compared to the "base" model.

\section{Discussion and Conclusions}

In urban areas, car use is often hindered by parking problems and congestion. In addition, good alternatives such as public transit and cycling are often existing. Nevertheless, many people own a car and use it regularly. With this study, we considered not only hard factors such as age, gender and income, but also soft factors such as the instrumental and affective motives for using a car to investigate their impacts on car use frequency. To uncover the different effects, we used an ordered hybrid choice model (OHCM) with a probit kernel, which allows us to consider hard as well as soft factors in the model parallelly. In this section, we discuss the main findings of our study and their implications for policy and planning and address various aspects of our holistic approach.

Regarding sociodemographic characteristics, the influence of age on the frequency of use has to be emphasized. People over the age of 30 were more likely to use a car more often. However, this could not be attributed to the affective motive. We conclude that in both cities no age effect based on the affective motive can be observed. Young people did not use the car less often than older people. We suggest that this is not determined by an aversion to driving but rather because they do not need a car in their current life situation. For transport planning, this results in a constant demand of car travel, as a flattening of use in the near future due to the aging of the younger generation cannot be expected. Further, the availability of a car in the household was a significant aspect regarding car use frequency. Individuals with permanent access to a car used it more frequently. This effect was strengthened by premium cars in the household, what is also confirmed by Niklas et al. [40]. Furthermore, in Berlin, for example, car ownership is promoted by a privileged residential parking ( $€ 10$ per year). Based on this people have no financial motivation to abandon their cars beyond the maintenance costs. It may be an option for policy makers to rise parking costs even for residents to initiate people's decision making process regarding car ownership. A further important policy measure that can be derived from this is the reduction of car ownership through various alternatives such as premium car sharing. For this purpose, pilot projects such as "Neue Mobilität Berlin" with their implemented "summer fleet" have been launched [41]. A comparable project example dealing with the results of breaking habitual car use for a defined period of time is provided by Sucha et al. [42]. Their results support the hypotheses and policy recommendations written above: financial aspects are not the main factor that lead to an abandonment of cars. Much more the aspect of comfort has to be focused. For example, in form of the difficulty to find a parking spot or being flexible enough with other modes of transport than the car. The perceived comfort of car use is still a relevant aspect for car travel. If, first, public transit can achieve the status as convenient and time-saving mode of transportation and, second, people experience this, a change in habitual behavior may be possible. For this reason, practical projects like this can support people who are thinking about reducing their car use in the long-term implementation of a car-free lifestyle. All above, a socially fair pricing of roads or parking in the city centers can reduce car use and relieve the roads. An additional and highly relevant finding in our study was that almost 
no difference between people from Berlin and San Francisco could be determined, certainly not among car users. This can be explained by the similar mobility culture in both cities. People from Berlin had a higher influence on instrumental motives than people from San Francisco (see Table 4B), but this had only a very low effect on car use frequency.

When considering long-distance travel, we found that more local-oriented people were more likely to use a car in everyday travel. Their behavior was characterized with many daytrips, such as visits or leisure activities outside the environment they are moving usually. In contrast, people who performed longer trips with overnight stays were less likely to use a car for everyday travel. They did not need a car in everyday situations, but only for occasional events in long-distance travel. Attractive car rental offers could reduce car ownership in this case because some of them have a car just for long-distance travels with overnight stays such as visiting parents in another city.

With respects to spatial effects, we found that a good public transit service reduced the use frequency of cars. As a result, a well-operating public transit system is the minimum requirement for cities to solve their traffic problems and to reduce emissions. This insight is generally not new. However, the results in the OHCM show the differences in terms of accessibility, which also exist in the two modern cities. Not all persons have the same access in the urban area, and we suggest that this has also an immediate effect on car use in modern cities. In contrast to existing studies where urban, suburban or rural areas have been compared with each other, we found that differences were also visible in urban areas.

As the main focus of our work concerned the influence of motives on car use, we summarize the essential results in this section. The indirect effects through the latent variables (LV) demonstrated that only the affective motives had an influence and increased the probability of car use. For gender, car ownership, premium car and income, the affective motive significantly increased the probability to use a car more frequently. This also led to people on lower incomes driving a car more frequently if they had an emotional connection to it. Results thus demonstrate that affective motives play a decisive role in the frequency of car use in modern hybrid cities. These cities are already at a stage that many cities would like to reach, as they dispose a large share of public transit and non-motorized private modes. Nevertheless, the affective motive had a strong influence on car use in those cities. Even in modern cities, we found that the car is much more than just a means to an end. We suggest that the use of the car is still associated with strong emotions, even if daily experiences give no indication of these feelings due to congestion and parking problems. We did not observe car captives who used the car frequently due to a lack of alternatives. The lack of influence of an instrumental motive allows for different interpretations. The strongest argument are the transport alternatives in hybrid cities. People can switch to other transport modes in many situation if they do not want to drive a car. This reduces the car captives in the city in contrast to cities such as Los Angeles (U.S.). Another argument are the car use conditions in the cities. Instrumental motives are not sufficient to encourage more frequent car use than necessary, as congestion and parking problems reduce the benefits of cars. People from Berlin regarded the car more as an instrument, but this did not lead to a different car use compared to people from San Francisco. This indicates again that the use frequency is detached from the instrumental motive in cities. There may be uses from instrumental motives, but these do not lead to more or less frequent use. A shortcoming of our approach is the low, but acceptable reliability of the factor (instrumental motive) as mentioned in Section 4. In future studies, additional items would be helpful for measuring the instrumental motive.

However, results suggest that the high affective motive complicates the substitution by transport alternatives in urban area. Car-loving users with affective motives are more likely to change their choice of destination if possible than to change their means of transport. As a result, only restrictions like dynamic and socially fair pricing of parking management or congestion charges could reduce car use frequency. Driving bans can further aggravate the situation, as public transit systems are unable to satisfy the capacity requirements in peak hours. Moreover, this can lead to a shift of trips to more distant destinations by car-loving users (e.g., shopping in suburban areas). Furthermore, 
origin destination relations have to be more intensively considered in the offer of public transit services if this should become an attractive alternative to cars [42,43]. A local reduction in emissions can be achieved through electric mobility in cities. This would not mean that the number of cars will decrease but managing private transport will become more environmentally friendly. In conclusion, the car is still relevant, also in modern hybrid cities.

As emotional factors contribute to the frequency of car use they may also affect habitual behavior. Domarchi et al. [44] mentioned that if positive attitudes to the car exist, it is possible that car use habits are strengthened. In addition, Chen and Chao [45] found that the intention and resistance to switch from car to public transit is particularly hindered for habitual car users. This was also confirmed by Nguyen et al. [46]. Building up on the assumption that frequent car use is related to habit, we can conclude that people's positive affective motive can promote this behavior. Consequently, the chain of action can be designed in such a way that positive emotions trigger car use, which in turn results in habitual actions. This will make it more difficult for transport policy as habits are not easy to change. We further see the importance of such soft attributes concerning the choice of modes. The difference regarding the perceived attractiveness of modes such as between car and public transit has to be reduced [3]. With this and with the aim to influence travel behavior especially emotional factors have to be considered. From this we can draw further implications for policy makers: if public transit is able to response to affective values of people, it will become a more preferred mode of transport.

At this point, we additionally would like to mention that the data were collected before the current pandemic. In the present situation, public transit use has decreased and a high impact on the intention to use public transit has become visible. This can lead to a reduction of the effect of public transit availability in our model. However, the question remains to what extent the current situation will affect future habits. This potential change may also have an impact on the influence of motives on car use and, for example, reinforce affective motives.

Further research can be conducted under inclusion of the psychological factor "autonomy" into the models. Autonomy describes whether people, from their point of view, assess it as possible to fulfill their mobility needs with another mode than a car. This addresses the consumed perception of car drivers regarding available alternatives. A comparison with another city with a different mobility culture such as more car-oriented cities (e.g., Los Angeles) would also provide more differentiated insights.

Author Contributions: Conceptualization, S.v.B.; methodology, S.v.B.; software, S.v.B.; validation, S.v.B., L.B., U.N. and B.C.; formal analysis, S.v.B.; investigation, S.v.B., L.B., U.N. and B.C.; resources, B.C.; writing-original draft preparation, S.v.B., L.B. and U.N.; writing-review and editing, S.v.B., L.B., U.N. and B.C.; visualization, S.v.B.; supervision, B.C.; project administration, S.v.B. and U.N.; funding acquisition, B.C. All authors have read and agreed to the published version of the manuscript.

Funding: This research received no external funding.

Acknowledgments: This study presents results based on the data of the project Urban Travel Monitor (UTM) funded by BMW AG. The anonymous reviewers helped to enhance the quality of this study and its outcome. We acknowledge support by the KIT-Publication Fund of the Karlsruhe Institute of Technology.

Conflicts of Interest: The authors declare no conflicts of interest.

\section{References}

1. Ecke, L.; Chlond, B.; Magdolen, M.; Eisenmann, C.; Hilgert, T.; Vortisch, P. Deutsches Mobilitätspanel (MOP)_Wissenschaftliche Begleitung und Auswertungen Bericht 2017/2018: Alltagsmobilität und Fahrleistung; Karlsruher Institut für Technologie, Institut für Verkehrswesen: Karlsruhe, Germany, 2019.

2. infas; DLR; IVT Research; infas 360. Mobilität in Deutschland-Ergebnisbericht. 2017. Available online: http://www.mobilitaet-in-deutschland.de/pdf/MiD2017_Ergebnisbericht.pdf (accessed on 27 June 2020).

3. Alteneder, W.; Risser, R. Soziologie der Verkehrsmittelwahl: Motive und Bedürfnisse im Zusammenhang mit der Verkehrsmittelwahl. Z. Verk. 1995, 41, 77-83.

4. Steg, L.; Vlek, C.; Slotegraaf, G. Instrumental-reasoned and symbolic-affective motives for using a motor car. Transp. Res. Part F4 2001, 4, 151-169. [CrossRef] 
5. Gardner, B.; Abraham, C. Psychological correlates of car use: A meta-analysis. Transp. Res. Part F Traffic Psychol. Behav. 2008, 11,300-311. [CrossRef]

6. Steg, L. Car use: Lust and must. Instrumental, symbolic and affective motives for car use. Transp. Res. Part A Policy Pract. 2005, 39, 147-162. [CrossRef]

7. Hunecke, M. Mobilitätsverhalten Verstehen und Verändern: Psychologische Beiträge zur Interdiszipliniaren Mobilitätsforschung; Springer: Berlin, Germany, 2015.

8. Bergstad, C.J.; Gamble, A.; Hagman, O.; Polk, M.; Gärling, T.; Olsson, L.E. Affective-symbolic and instrumental-independence psychological motives mediating effects of socio-demographic variables on daily car use. J. Transp. Geogr. 2011, 19, 33-38. [CrossRef]

9. Belgiawan, P.F.; Schmöcker, J.-D.; Abou-Zeid, M.; Walker, J.; Lee, T.-C.; Ettema, D.F.; Fujii, S. Car ownership motivations among undergraduate students in China, Indonesia, Japan, Lebanon, Netherlands, Taiwan, and USA. Transportation 2014, 41, 1227-1244. [CrossRef]

10. Ellaway, A.; Macintyre, S.; Hiscock, R.; Kearns, A. In the driving seat: Psychosocial benefits from private motor vehicle transport compared to public transport. Transp. Res. Part F Traffic Psychol. Behav. 2003, 6, 217-231. [CrossRef]

11. Shiftan, Y.; Outwater, M.L.; Zhou, Y. Transit market research using structural equation modeling and attitudinal market segmentation. Transp. Policy 2008, 15, 186-195. [CrossRef]

12. Anable, J. 'Complacent Car Addicts' or 'Aspiring Environmentalists'? Identifying travel behaviour segments using attitude theory. Transp. Policy 2005, 12, 65-78. [CrossRef]

13. Kamargianni, M.; Dubey, S.; Polydoropoulou, A.; Bhat, C. Investigating the subjective and objective factors influencing teenagers' school travel mode choice-An integrated choice and latent variable model. Transp. Res. Part A Policy Pract. 2015, 78, 473-488. [CrossRef]

14. Roberts, J.; Popli, G.; Harris, R.J. Do environmental concerns affect commuting choices? Hybrid choice modelling with household survey data. J. R. Stat. Soc. A 2018, 181, 299-320. [CrossRef]

15. Hunecke, M.; Haustein, S.; Böhler, S.; Grischkat, S. Attitude-Based Target Groups to Reduce the Ecological Impact of Daily Mobility Behavior. Environ. Behav. 2010, 42, 3-43. [CrossRef]

16. Lois, D.; López-Sáez, M. The relationship between instrumental, symbolic and affective factors as predictors of car use: A structural equation modeling approach. Transp. Res. Part A Policy Pract. 2009, 43, 790-799. [CrossRef]

17. Sohn, K.; Yun, J. Separation of car-dependent commuters from normal-choice riders in mode-choice analysis. Transportation 2009, 36, 423-436. [CrossRef]

18. Sefara, D.; Franek, M.; Zubr, V. Socio-psychological factors that influence car prefernce in undergraduate students: The case of the Czech Republic. Technol. Econ. Dev. Econ. 2015, 21, 643-659. [CrossRef]

19. Nalmpantis, D.; Roukouni, A.; Genitsaris, E.; Stamelou, A.; Naniopoulos, A. Evaluation of innovative ideas for Public Transport proposed by citizens using Multi-Criteria Decision Analysis (MCDA). Eur. Transp. Res. Rev. 2019, 11. [CrossRef]

20. Ashok, K.; Dillon, W.R.; Yuan, S. Extending Discrete Choice Models to Incorporate Attitudinal and Other Latent Variables. J. Mark. Res. 2002, 39, 31-46. [CrossRef]

21. Ben-Akiva, M.; Mcfadden, D.; Gärling, T.; Gopinath, D.; Walker, J.; Bolduc, D.; Börsch-Supan, A.; Delquié, P.; Larichev, O.; Morikawa, T.; et al. Extended Framework for Modeling Choice Behavior. Mark. Lett. 1999, 10, 187-203. [CrossRef]

22. Walker, J.L. Extended Discrete Choice Models: Integrated Framework, Flexible Error Structures, and Latent Variables. Ph.D. Thesis, Massachusetts Institute of Technology, Cambridge, MA, USA, 2001.

23. Nurul Habib, K.; Tudela, A.; Carrasco, J.; Idris, A. Incorporating the explicit role of psychological factors on mode choice: A hybrid mode choice model by using data from an innovative psychometric survey. In Proceedings of the Second International Choice Modeling Conference, Leeds, UK, 6 July 2011.

24. Yáñez, M.F.; Raveau, S.; Ortúzar, J.D.D. Inclusion of latent variables in Mixed Logit models: Modelling and forecasting. Transp. Res. Part A Policy Pract. 2010, 44, 744-753. [CrossRef]

25. Johansson, M.V.; Heldt, T.; Johansson, P. The effects of attitudes and personality traits on mode choice. Transp. Res. Part A Policy Pract. 2006, 40, 507-525. [CrossRef]

26. Abrahamse, W.; Steg, L.; Gifford, R.; Vlek, C. Factors influencing car use for commuting and the intention to reduce it: A question of self-interest or morality? Transp. Res. Part F Traffic Psychol. Behav. 2009, 12, 317-324. [CrossRef] 
27. Márquez, L.; Macea, L.F.; Soto, J.J. Willingness to change car use to commute to the UPTC main campus, Colombia: A hybrid discrete choice modeling approach. JTLU 2019, 12. [CrossRef]

28. De Vos, J.; Alemi, F. Are young adults car-loving urbanites? Comparing young and older adults' residential location choice, travel behavior and attitudes. Transp. Res. Part A Policy Pract. 2020, 132, 986-998. [CrossRef]

29. Van, H.T.; Fujii, S. A cross Asian country analysis in attitudes toward car and public transport. J. East. Asia Soc. Transp. Stud. 2011, 9, 411-421.

30. Institute for Mobility Research. Urban Mobility in China. 2017. Available online: https://www.bmwgroup.com/content/dam/grpw/websites/bmwgroup_com/company/downloads/ de/2017/2017-BMW-Group-IFMO-Publikation-Juni.pdf (accessed on 27 June 2020).

31. von Behren, S.; Minster, C.; Magdolen, M.; Chlond, B.; Hunecke, M.; Vortisch, P. Bringing travel behavior and attitudes together: An integrated survey approach for clustering urban mobility types. In Proceedings of the 97th Annual Meeting of the Transportation Research Board, Washington, DC, USA, 7-11 January 2018.

32. von Behren, S.; Minster, C.; Esch, J.; Hunecke, M.; Vortisch, P.; Chlond, B. Assessing car dependence: Development of a comprehensive survey approach based on the concept of a travel skeleton. Transp. Res. Procedia 2018, 32, 607-616. [CrossRef]

33. Kuhnimhof, T.; Wulfhorst, G. The Reader's Guide to Mobility Culture. Chapter 3. In Megacity Mobility Culture-How Cities Move on in a Diverse World; Institute for Mobility Research, Ed.; Springer: Berlin/Heidelberg, Germany, 2013; pp. 55-64. ISBN 978-3-642-34734-4.

34. Klinger, T.; Lanzendorf, M. Moving between mobility cultures: What affects the travel behavior of new residents? Transportation 2015, 43, 243-271. [CrossRef]

35. San Francisco Municipal Transportation Agency. Travel Decisions Survey. Summary Report. 2017. Available online: https:/www.sfmta.com/sites/default/files/reports/2017/Travel\%20Decisions\%20Survey\% 20Summary\%20Report\%202017_Accessible.pdf (accessed on 27 June 2020).

36. Senatsverwaltung für Umwelt, Verkehr und Klimaschutz. Mobilität der Stadt. Berliner Verkehr in Zahlen. 2017. Available online: https://www.berlin.de/senuvk/verkehr/politik_planung/zahlen_fakten/download/ Mobilitaet_dt_komplett.pdf (accessed on 27 June 2020).

37. Bhat, C.R.; Dubey, S.K. A new estimation approach to integrate latent psychological constructs in choice modeling. Transp. Res. Part B Methodol. 2014, 67, 68-85. [CrossRef]

38. Vij, A.; Walker, J.L. How, when and why integrated choice and latent variable models are latently useful. Transp. Res. Part B Methodol. 2016, 90, 192-217. [CrossRef]

39. CMC. CMC Choice Modelling Code for R; Choice Modelling Centre, University of Leeds: Leeds, UK, 2017.

40. Niklas, U.; von Behren, S.; Eisenmann, C.; Chlond, B.; Vortisch, P. Premium factor-Analyzing usage of premium cars compared to conventional cars. Res. Transp. Bus. Manag. 2020, 25, 100456. [CrossRef]

41. Neue Mobilität Berlin. NEUE MOBILITÄT BERLIN-Innovative Mobilitätsentwürfe für Berlin. Available online: http://neue-mobilitaet.berlin/?lang=en (accessed on 6 March 2020).

42. Sucha, M.; Viktorova, L.; Risser, R. Can an Experience with No Car Use Change Future Mode Choice Behavior? Sustainability 2019, 11, 4698. [CrossRef]

43. Esztergár-Kiss, D.; Koppányi, Z.; Lovas, T. Mobility Mapping Based on a Survey from the City of Berlin. Period. Polytech. Transp. Eng. 2016, 44, 35-41. [CrossRef]

44. Domarchi, C.; Tudela, A.; González, A. Effect of attitudes, habit and affective appraisal on mode choice: An application to university workers. Transportation 2008, 35, 585-599. [CrossRef]

45. Chen, C.-F.; Chao, W.-H. Habitual or reasoned? Using the theory of planned behavior, technology acceptance model, and habit to examine switching intentions toward public transit. Transp. Res. Part F Traffic Psychol. Behav. 2011, 14, 128-137. [CrossRef]

46. Nguyen, N.T.; Miwa, T.; Morikawa, T. Switching to Public Transport Modes for Commuting Trips: Considering Latent Motivations in Ho Chi Minh City. Asian Transp. Stud. 2018, 5, 117-136. [CrossRef]

(C) 2020 by the authors. Licensee MDPI, Basel, Switzerland. This article is an open access article distributed under the terms and conditions of the Creative Commons Attribution (CC BY) license (http://creativecommons.org/licenses/by/4.0/). 